\title{
Recognizing and managing anxiety disorders in primary health care in Turkey
}

\author{
Mehtap Kartal*+1, Ozlem Coskun ${ }^{\dagger 2}$ and Nesrin Dilbaz ${ }^{\dagger 3}$
}

\begin{abstract}
Background: Anxiety disorders are common and are frequently not diagnosed accurately in primary care. Our aim was to determine the knowledge gaps of general practitioners (GPS) in the diagnosis and treatment of anxiety disorders by using vignettes.

Methods: A cross-sectional survey was completed with 255 primary care physicians (response rate 59.4\%) in Manisa, a city in western Turkey. From the postal questionnaire, information on working experience, postgraduate education in psychiatry, the interests of the physicians in psychiatry were obtained. The physicians' diagnosis and treatment preferences for generalized anxiety disorder (GAD), social phobia (SP), and obsessive compulsive disorder (OCD) were determined through clinical vignettes prepared for data collection.

Results: Two hundred and twenty-seven (89.0\%) out of 255 GPs included the diagnosis of obsessive compulsive disorder in their differential diagnosis; however, the rates for social phobia and generalized anxiety disorder were 69.4\% $(n=177)$ and $22.3 \%(n=57)$, respectively. GPs with a post-graduate education on psychiatry diagnosed vignettes more accurately for OCD $(p=0.04)$. For all three cases, GPs mostly preferred a combination therapy including psychotherapy and psycho-pharmacotherapy. The referral rate to a psychiatrist was between 23.1 and 30.6\%. The percentages of the prescription of selective serotonin reuptake inhibitors (SSRI) in accurate diagnosis were 59.3 for social phobia, 33.3 for GAD, and 55.5 for OCD.
\end{abstract}

Conclusions: There is a gap of knowledge in GPs, which leads to poor recognition and management of anxiety disorders in primary care. Effective interventions including post-graduate education and updated guidelines on anxiety disorders should be planned and implemented with their assessments by vignettes.

\section{Background}

Anxiety disorders are the most common disorders with a 12-month prevalence changing between 2.4 and $18.2 \%$ among the general population [1]. For the subgroups of anxiety disorders, the prevalence is $0.6-5.2 \%$ for social phobia (SP), $0.5-3.8 \%$ for generalized anxiety disorder (GAD), and $0.3-4.0 \%$ for obsessive compulsive disorder (OCD) [2-5].

Patients with anxiety disorders increasingly consult primary care units and this is reflected in the prevalence of anxiety disorders (14.6-19.0\%), social phobia (2.6-12.3\%), GAD (2.8-13.2\%), and OCD (2.0\%) in primary care [6-10].

\footnotetext{
*Correspondence: mehtapkartal@gmail.com

1 Family Medicine Department of Dokuz Eylul University, 35340, Inciralti, Izmir, Turkey

+ Contributed equally

Full list of author information is available at the end of the article
}

In Turkey, although we do not have population-based prevalence studies for subgroups of mental disorders such as anxiety disorders, we know that more than $17 \%$ of the adult population has certain mental health problems. The National Burden of Disease Study results revealed that unipolar depressive disorder was ranked seven in the list of national disability-adjusted life year (DALY) calculations for the first 20 diseases [11,12]. It was estimated that only $13 \%$ of all the mentally ill patients could receive help for their problems [11].

Approximately $12-22 \%$ of the primary care patients present with symptoms of distress related to anxiety. General practitioners (GPs) identify about $50 \%$ of psychiatric disorders, and only one third of anxiety cases are correctly diagnosed $[11,13]$. The rate of accurate specific diagnoses for anxiety disorder is $35-65 \%$, for pure GAD $34.4 \%$, and for social phobia $24.0 \%[14,15]$. These low rates may be due to patient-related reasons (such as unex- 
pressed psychological problems, normalizing the symptoms, and the fear of stigmatization of mental illness), or physician-related reasons (such as unawareness of the diagnosis, inadequate recognition, competing demands in a limited time) [15-17]. Only $25 \%$ of the primary care patients with anxiety disorders received adequate medication, and less than $10 \%$ of them were counseled by a mental health professional [18].

In Turkey, undergraduate medical education starts after the university entrance examination following the secondary school, and lasts for 6 years. A basic doctor after graduation may practice in primary care as there is no necessity of residency or fellowship for implementation of practice. GPs work nearly in all health facilities including primary care centers, tuberculosis (TB) prevention dispensaries, maternal and child health centers, and the emergency services of state hospitals. There is no referral system from primary care, so patients can either be referred by their physicians in primary care or they can directly apply to all the hospitals, including those for mental health. Specialization in "Family Medicine" is still in its developing period with nearly 1,500 family medicine specialists working for health care.

The aim of this study was to examine the GPs' knowledge of anxiety disorders (social phobia, generalized anxiety disorder, and obsessive compulsive disorder) by clinical vignettes which are commonly used in the studies of depression and psychosis, and to our knowledge, which have not been done with anxiety disorders before.

\section{Methods}

We conducted a postal survey for all GPs working in primary care institutions of the Ministry of Health (primary health care centers, maternal and child health centers, and dispensaries) in Manisa. Manisa is a city located in the Aegean region, with a population of 1,267,493 (Male 634,557, Female 632,936), $74.8 \%$ of which live in urban areas. In 2005, the health services in the city included 158 primary health care centers, 6 TB prevention dispensaries, 4 maternal and child health centers, 16 state hospitals, one children's hospital, one maternal hospital, and one mental health hospital. The mental health hospital is one of the oldest health facilities with a history beginning at 1539. It serves a total of 12 cities in the Aegean and West Mediterranean region with 400 beds.

As we do not have a specific questionnaire to assess GPs' knowledge in the diagnosis and management of anxiety disorders, we constructed a vignette-based instrument. Vignettes reflect a primary care presentation fulfilling Diagnostic and Statistical Manual of Mental Disorders, 4th edition (DSM-IV) diagnostic criteria for social phobia, generalized anxiety disorder, and obsessive compulsive disorder. The vignettes were assessed by three psychiatrists and finalized by a fourth one. The pilot study was conducted with ten psychiatrists and all the vignettes were diagnosed accurately. We also gathered additional information about the associated factors like working experience, postgraduate education on psychiatry and interest in psychiatry (Additional file 1).

The questionnaires and cover letters were posted to the sample in early January 2005 and were collected back in a month.

\section{Coding and analysis}

GPs were asked to write a brief description of their treatment plan in an open-ended format for SP, GAD, and OCD. The management options were assessed in five categories: psychotherapeutic support and/or watchful waiting, referral to a psychiatrist, psychotherapy, psychopharmacotherapy, and combined therapy (psychotherapy and psycho-pharmacotherapy). GPs also had a paper for prescription in case they preferred to fill for the patient. However, as there was no information as to the duration of the medical treatment, we only assessed the accuracy of the prescriptions in three categories: accurate, acceptable, and unacceptable treatment according to the Diagnosis and Treatment Guideline for Primary Care, 2003[19]. The first-line treatment was named as accurate, the treatments other than the first-line were named as acceptable; however, the time period of the treatments could not be evaluated as they were not mentioned in the prescriptions. The agreement between two independent raters for the evaluation of prescriptions in line with the Diagnosis and Treatment Guideline for Primary Care, 2003, was found as $86.7 \%$ for SP, $78.5 \%$ for GAD, and 97.3\% for OCD.

The data were installed and analyzed by the SPSS software, using descriptive statistics for frequencies, chisquare tests for the comparisons of independent proportions, with Fisher's exact test where appropriate. All the tests were two-tailed. The level of significance was set as $\mathrm{p}<0.05$. The prescriptions were reevaluated by two independent raters to report the agreement in terms of kappas.

\section{Results}

The response rate was $59.4 \%$ (255 out of 429). Of the participants, $145(56.9 \%)$ were male and $110(43.1 \%)$ were female. The mean age was $36.6 \pm 6.3$ years; age was grouped as 26-35 (42.0\%), 36-45(49.0\%), and 46-55 (9.0\%). The mean working experience was $12.3 \pm 6.0$ years, and $49.8 \%$ of the participants had a working experience $11-20$ years, $40.4 \%$ were in group of $1-10$ years. $42.7 \%$ of the participants mentioned that they had a postgraduate education on psychiatry, and $37.6 \%$ stated that they had an interest in psychiatry. 


\section{Diagnosis}

Two hundred and twenty-seven (89.0\%) GPs included obsessive compulsive disorder in their differential diagnosis of OCD; however, the rate was $69.4 \%(n=177)$ and $22.3 \%(n=57)$ respectively for social phobia and generalized anxiety disorder. Other common diagnoses for OCD were psychosis $(\mathrm{n}=5,2.0 \%)$, paranoid disorder $(\mathrm{n}=4$, $1.6 \%)$, anxiety ( $\mathrm{n}=2,0,8 \%)$; for SP they were antisocial personality disorder $(n=15,6.0 \%)$, anxiety $(n=14,5.6 \%)$, panic attack $(\mathrm{n}=11,4.4 \%)$; and for GAD they were panic disorder ( $n=93,37.8 \%)$, depression $(n=40,16.3 \%)$ and post traumatic stress disorder $(n=21,8.5 \%)$. Of all the GPs, 3.9\% ( $\mathrm{n}=10)$ diagnosed all vignettes inaccurately, $25.9 \%(n=66)$ diagnosed one vignette accurately, $55.7 \%$ $(\mathrm{n}=142)$ diagnosed two vignettes accurately and only $14.5 \%(n=37)$ diagnosed all the vignettes accurately.

There were no significant differences between the gender, age and interest in psychiatry of the GPs and the accurate diagnosis of anxiety disorders $(p>0.05)$. The GPs with a post-graduate education (97.1\%) on psychiatry accurately diagnosed the OCD vignettes more frequently than those with no post-graduate education $(90.0 \%)$ on psychiatry $(\mathrm{p}=0.04)$.

\section{Treatment and Referrals}

The treatment preferences of the GPs for the vignettes, according to the accuracy of their diagnosis, were shown in Figure 1. For all three vignettes diagnosed accurately, the GPs mostly preferred a combination therapy including psychotherapy and psycho-pharmacotherapy. There was no significant relationship between the gender and the age of the GPs and the treatment options ( $p>0.05$ ). The referral rates of the male GPs were $29.0 \%$ and $36.6 \%$ for GAD ( $\mathrm{p}=0.007)$ and OCD $(\mathrm{p}=0.02)$, respectively, which were higher than those of the female GPs $(14.5 \%$ and $22.7 \%$ ). The GPs with a post-graduate education and interest in psychiatry prefer referral for OCD (20.2\%; $17.7 \%)$, which were lower than those with no education (38.4\%; $\mathrm{p}=0.002$ ) and interest (38.4\%; $\mathrm{p}=0.0001)$. For GAD, this is also true with percentages of $8.3 \%$ and $33.6 \%$ for post-graduate education $(\mathrm{p}=0.0001)$ and $15.6 \%$ and $27.0 \%$ for interest in psychiatry $(\mathrm{p}=0.045)$, respectively.

The GPs with accurate diagnoses (25.6\%) were less likely to refer the patient compared to those GPs with inaccurate diagnoses (64.7\%) for OCD vignette ( $p=$ $0.001)$, who preferred the referral more.

\section{Prescribing}

In SP, GAD and OCD vignettes, the GPs preferred not to prescribe any drugs with a rate of $33.3 \%, 27.8 \%$, and $37.6 \%$, respectively, and they preferred psychotherapeutic support and/or watchful waiting or referral to a psychiatrist or psychotherapy (Table 1).
For all anxiety cases, selective serotonin reuptake inhibitors (SSRI) were the first-line drugs preferred by the GPs. The accuracy of prescriptions were evaluated as accurate, acceptable and unacceptable treatment according to the Diagnosis and Treatment Guideline for Primary Care, 2003 , and the accuracy rates for SP, GAD, and OCD were $59.3 \%, 33.3 \%$, and $55.5 \%$, respectively (Figure 2). Out of 37 GPs who diagnosed all the vignettes accurately, only 10 prescribed appropriate treatment.

The percentages of the GPs who preferred to prescribe more than one medication were $19.6 \%, 35.7 \%$, and $12.9 \%$ for SP, GAD, and OCD, respectively. The most commonly seen combinations were SSRI+benzodiazepine (10 GPs), $\mathrm{MAO}$ inh+MAO inh (7 GPs) for SP, SSRI+benzodiazepine (21 GPs), SSRI+B bloker (6GPs) for GAD and SSRI+TCA (11 GPs) and SSRI+benzodiazepine (7 GPs) for OCD.

\section{Discussion and Conclusion Summary of main findings}

Our vignettes for OCD and SP were diagnosed accurately by $89.0 \%$ and $69.4 \%$ of the GPs, respectively suggesting that the GPs were aware of these patients; however, they had difficulty in the diagnosis of GAD (22.4\%). Although we portrayed our vignettes according to the DSM-IV diagnostic criteria, there can be a point that might have led the GPs to misdiagnosis. However, this factor alone cannot adequately explain why the accuracy of the diagnosis for GAD was so low. This may be due to the high co-morbidity such as depression or any other anxiety disorder which could have been misdiagnosed as panic disorder and depression [4,6,18-21]. Our rates were similar to those of other studies $[10,15]$. The natural history of GAD being chronic, its controversial nosologic validity over the past 20 years, and the relatively recent interest in its independent impact and treatment in primary care may have contributed to its low rate of recognition $[8,10,22]$. In daily practice, patients also have a great impact on under-recognition and misdiagnosis of anxiety disorders; however, this will not be discussed here as we used vignettes.

The most commonly used treatment options by primary care providers are pharmacotherapy and psychotherapy $[7,22]$. While psycho-pharmacotherapy alone and psychotherapy alone were chosen by $13.7-22.4 \%$ and 3.9 $6.7 \%$ of the GPs, respectively, their combination was preferred by $47.5-50.6 \%$ of them. In a study conducted in 1997 and 1998, psycho-pharmacotherapy alone and psychotherapy alone were chosen in $54.6 \%$ and $2.0 \%$ of visits to primary care for anxiety disorders, respectively [23]. However, in a recent study, psycho-pharmacotherapy alone, and psychotherapy alone were used by $21.0 \%$ and $7.2 \%$ of the GPs, respectively [24]. The rates for the com- 

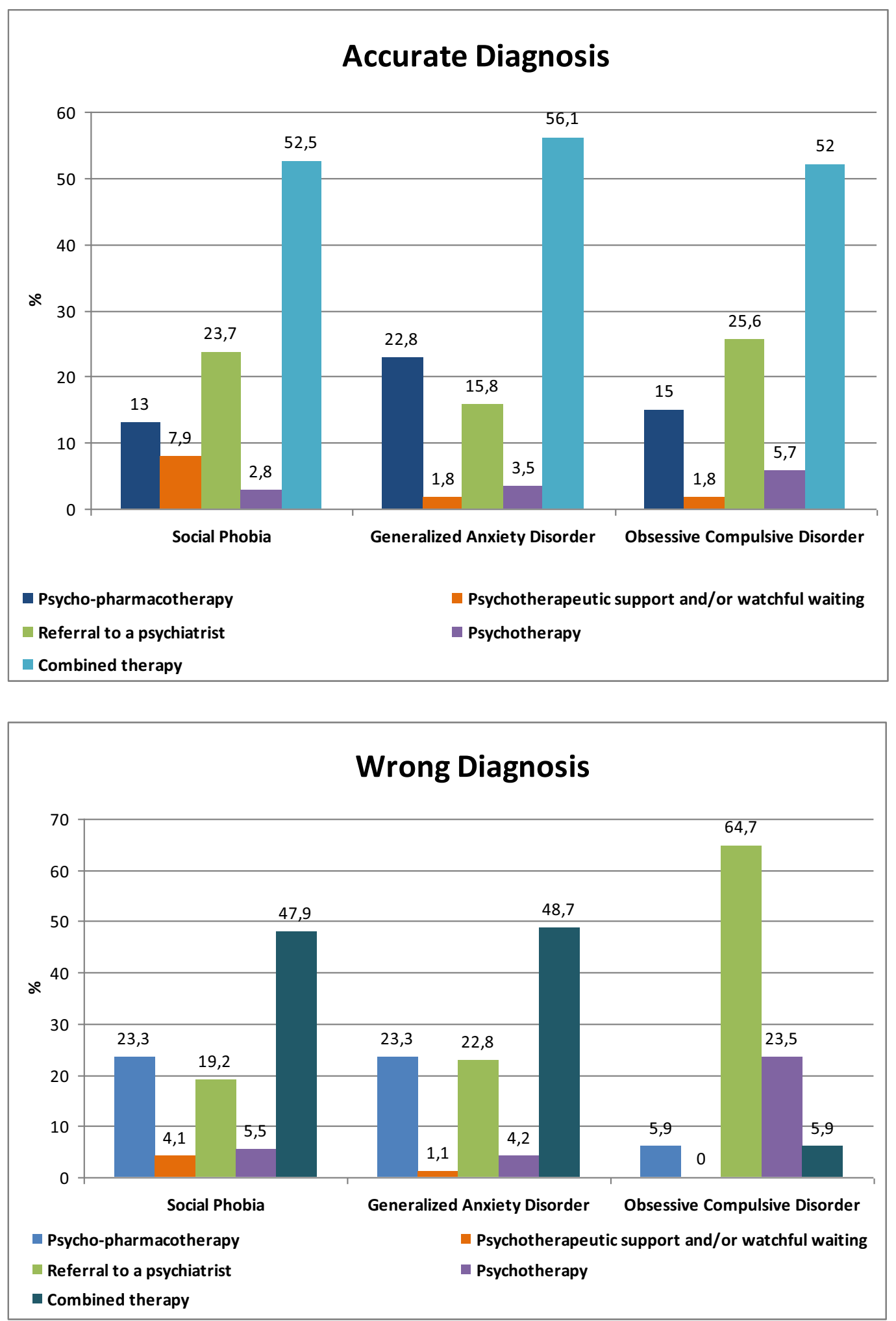

Figure $1 \mathrm{GPs}$ ' treatment choices for the vignettes according to the accuracy of the diagnosis 
Table 1: The treatment preferences of GPs for anxiety disorders

\begin{tabular}{|c|c|c|c|}
\hline & $\begin{array}{l}\text { Social Phobia } \\
\% \text { (n) }\end{array}$ & $\begin{array}{l}\text { Generalized Anxiety } \\
\text { Disorder } \\
\%(n)\end{array}$ & $\begin{array}{l}\text { Obsessive Compulsive } \\
\text { Disorder } \\
\%(n)\end{array}$ \\
\hline No prescription & $33.3(85)$ & $27.8(71)$ & $37.6(96)$ \\
\hline $\begin{array}{l}\text { Psychotherapeutic } \\
\text { support and/or watchful } \\
\text { waiting }\end{array}$ & $18.8(16)$ & $2.8(2)$ & $2.1(2)$ \\
\hline Referral to a psychiatrist & $69.4(59)$ & $81.7(58)$ & $81.3(78)$ \\
\hline Psychotherapy & $11.8(10)$ & $15.5(11)$ & $16.7(16)$ \\
\hline Prescription & $66.7(170)$ & $72.2(184)$ & $62.4(159)$ \\
\hline \multicolumn{4}{|l|}{ Prescribed drugs* } \\
\hline & $\%\left(n^{a}\right)$ & $\%\left(n^{b}\right)$ & $\%(n c)$ \\
\hline SSRI & $43.5(97)$ & $48.8(140)$ & $62.5(120)$ \\
\hline Benzodiazepines & $8.5(19)$ & $23.3(67)$ & $6.3(12)$ \\
\hline TCA & $7.6(17)$ & $6.3(18)$ & $25.5(49)$ \\
\hline MAO inhibitors & $21.5(48)$ & $1.0(3)$ & $0.5(1)$ \\
\hline B blocker & $6.7(15)$ & $4.9(14)$ & $0.0(0)$ \\
\hline SNRI & $2.2(5)$ & $3.1(9)$ & $2.1(4)$ \\
\hline Antipsychotic & $0.0(0)$ & $2.4(7)$ & $0.5(1)$ \\
\hline Others & $9.9(22)$ & $10.1(29)$ & $2.6(5)$ \\
\hline
\end{tabular}

Number of drugs prescribed: ${ }^{a} n=223 .{ }^{b} n=287 .{ }^{c} n=192$.

* More than one drug is prescribed

bination treatment had a wide range; $2.8 \%$ in one study and $24.5 \%$ in another $[23,24]$. In spite of the difficulties of the interpretation due to methodological differences, it can be commented that there is a tendency towards the combination therapy, and pharmacotherapy has much more impact than psychotherapy, which was also supported by our study [10].

SSRIs are typically considered as the first-line pharmacotherapy in treating patients with anxiety disorders both in secondary and primary care $[7,19,24]$. In primary care, the study results showed that the prescription of psychotropic drugs and their types do not always reflect the context of a specific diagnosis [13]. In our study, for all the vignettes with different percentages, the GPs preferred SSRIs in the first line. This finding supports the literature also. The percentage of adequate treatment by GPs was $20.9 \%$ for any anxiety disorder and $14.8 \%$ for GAD in Spain [25]. There is a need for improving the treatment adequacy for anxiety disorders.

Stein et al. reported that primary care physicians recommended referral to a psychiatrist by $37.4 \%$ in any anxiety disorder, by $37.7 \%$ in GAD and by $36.9 \%$ in social phobia [18]. However, our rates were lower than these figures. The referral rates with wrong diagnosis were $19.2 \%$, $22.8 \%$, and $64.7 \%$ for social phobia, GAD, and OCD, respectively. The GPs' low referral rates suggested that there is a risk of lack of appropriate treatment in primary care for all anxiety cases, but especially for GAD patients. Although the rates of GPs with post-graduate education in psychiatry for accurate diagnosis were not higher, their accurate treatment rates were higher and referral rates were lower. Although we have no idea about the content of the post-graduate education, treatment was known better than the diagnosis. The post-graduate education, being a part of multifaceted interventions, should not only focus on treatment but also on diagnosis.

It is unlikely to improve the recognition and management of anxiety disorders in primary care by a single intervention. It needs multifaceted interventions including professional and organizational focus on the implementation of post-graduate education, supporting collaborative care with the implementation of guidelines, the integration of primary care and mental health care $[7,10,14,15,18]$. 


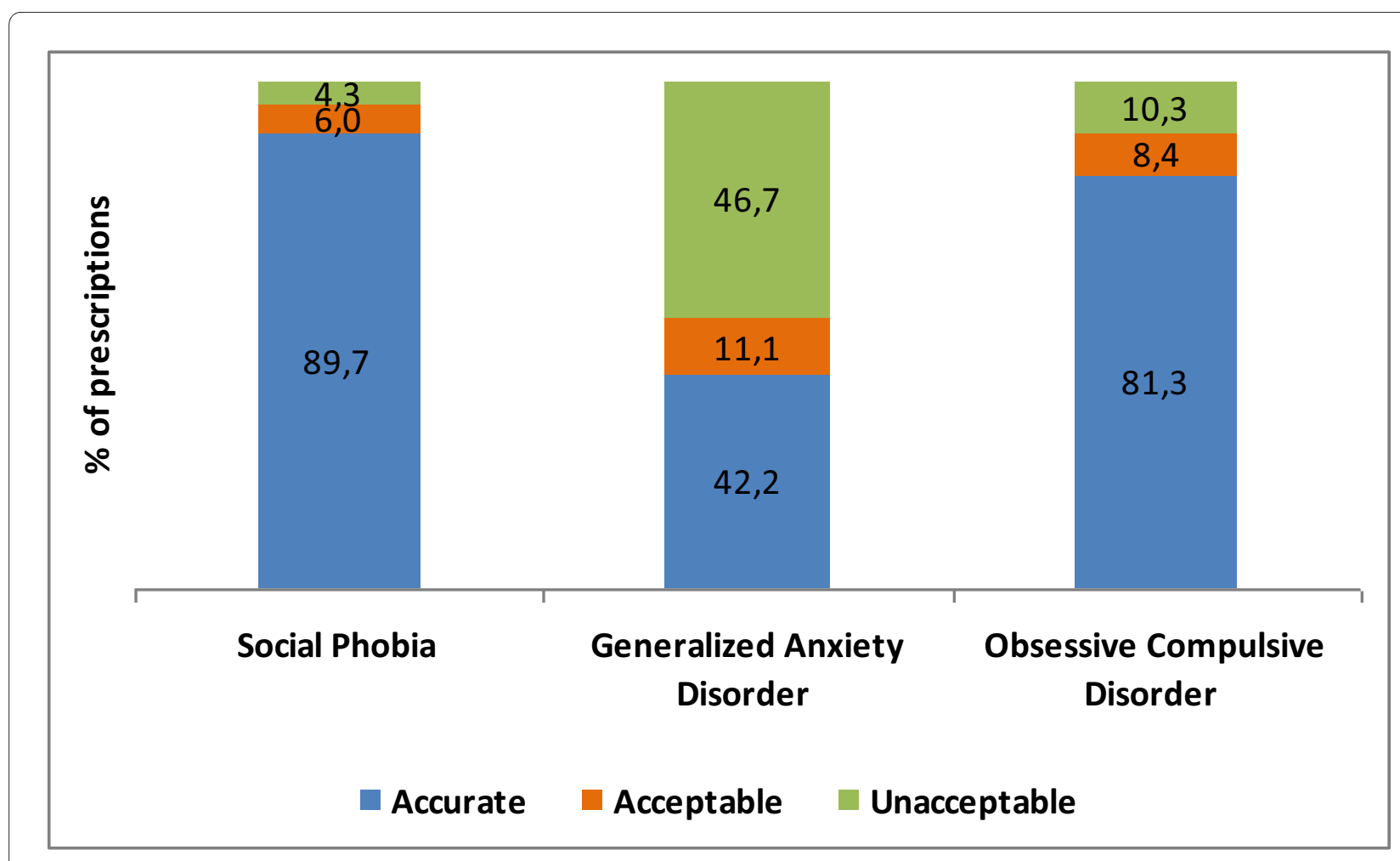

Figure $\mathbf{2}$ The evaluation of the prescriptions of GPs who diagnosed vignettes accurately.

\section{Strengths and limitations of the study}

Data collection by vignettes can be a method reflecting clinical practice. However, this method has its own limitations. Firstly, our vignettes reflect a primary care presentation fulfilling the DSM-IV diagnostic criteria and this was a necessity due to our guideline, in which the diagnosis of anxiety disorders is based on the DSM-IV. Secondly, vignettes, despite the criteria or guideline they are based on, have concerns about their validity in the exploration of professional decision making [26]. However, a limited number of recent studies support that they are suited for comparative analysis and more effective than chart abstracts $[27,28]$.

We have the limitation of risk for non-respondent bias with a completion rate of $59.4 \%$. We can not say that the respondents are representative of the original sample according to sex and age as we do not have these data. However, we think that this can also suggest an apparent lack of knowledge on anxiety disorders, and also that our results may reflect a bias towards better knowledge.

\section{Implications for clinical practice and future research}

Mental health problems, anxiety disorders in particular, are common in primary care. However, although they are as common as depression, they often receive less attention and they remain unrecognized and untreated [6-
10,13-15]. The high rates of co-morbidity with psychiatric disorders and physical illnesses result in varying and misleading presentations. The patients do not usually link their health issues to psychological problems. They even resist these diagnoses and show unwillingness to accept care. In daily practice, these factors coupled with limited time for interviews with patients conspire against GPs' accurate diagnosis of anxiety disorders [15-17]. For the quality of care and knowledge assessments in primary care, vignettes can be used more, especially in countries with limited data like Turkey.

It can be said that the results of our study are not surprising, considering the fact that anxiety disorders are not well-known and presented in practice. We believe that there is a need to question the low rates of accurate diagnosis for anxiety disorders. Effective interventions including post-graduate education and updated guidelines on anxiety disorders should be planned and implemented with their assessments by vignettes.

Competing interests

The authors declare that they have no competing interests.

\section{Authors' contributions}

MK participated in the design of the study and performed the statistical analysis. OC participated in its design and coordination and helped to draft the manuscript. All authors read and approved the final manuscript. 


\section{Acknowledgements}

We would like to thank the general practitioners in Manisa for their participation in the study.

\section{Author Details}

1 Family Medicine Department of Dokuz Eylul University, 35340, Inciralti, Izmir, Turkey, ${ }^{2}$ Medical Education Department of Gazi University, Ankara, Turkey and ${ }^{3}$ The First Psychiatry Clinic, Ankara Numune Education and Research Hospital, Ankara, Turkey

Received: 9 November 2009 Accepted: 28 April 2010

Published: 28 April 2010

\section{References}

1. The WHO World Mental Health Survey Consortium: Prevalence, severity, and unmet need for Treatment of Mental Disorders in the World Health Organization World Mental Health Surveys. JAMA 2004 291(21):2581-90.

2. Fehm L, Pelissolo A, Furmark T, Wittchen HU: Size and burden of social phobia in Europe. Eur Neuropsychopharmacol 2005, 15(4):425-34

3. Wittchen H-U, Hoyer J: Generalized Anxiety Disorder: Nature and Course. J Clin Psychiatry 2001, 62(Suppl 11):15-18.

4. Lieb R, Becker E, Altamura C: The epidemiology of generalized anxiety disorder in Europe. Eur Neuropsychopharmacol 2005, 15(4):445-52.

5. Fontenelle LF, Mendlowicz MV, Versiani M: The descriptive epidemiology of obsessive-compulsive disorder. Prog Neuropsychopharmacol Biol Psychiatry 2006, 30(3):327-37.

6. Nisenson LG, Pepper CM, Schwenk TL, Coyne JC: The nature and prevalence of anxiety disorders in primary care. Gen Hosp Psychiatry 1998, 20(1):21-28

7. Stein MB: Attending to anxiety disorders in primary care. J Clin Psychiatry 2003, 64(Suppl 15):35-39.

8. Ansseau M, Dierick M, Buntinkx F, Cnockaert P, De Smedt J, Haute M Van Den, Mijnsbrugge D Vander: High prevalence of mental disorders in primary care. J Affect Disord 2004, 78:49-55.

9. Kroenke K, Spitzer RL, Williams JB, Monahan PO, Löwe B: Anxiety disorders in primary care: prevalence, impairment, comorbidity, and detection. Ann Intern Med 2007, 146(5):317-25

10. Roy-Byrne PP, Wagner A: Primary care perspectives on generalized anxiety disorder. J Clin Psychiatry 2004, 65(Suppl 13):20-6.

11. Soykan A, Oncu B: Which GP deals better with depressed patients in primary care in Kastamonu, Turkey: the impacts of 'interest in psychiatry' and 'continuous medical education'. Fam Pract 2003, 20(5):558-62.

12. Ministry of Health, Refik Saydam Hygiene Center Presidency: National Burden of Disease and Cost-Effectiveness Project, Burden of Disease Final Report 2004 [http://www.hm.saglik.gov.tr/pdf/nbd/raporlar/ burdenofdiseaseENG.pdf]. Accessed July 2005

13. Lecrubier Y: Prescribing patterns for depression and anxiety worldwide. J Clin Psychiatry 2001, 68(Suppl 13):31-36.

14. Heideman J, van Rijswijk E, van Lin N, de Loos S, Laurant M, Wensing M, Lisdonk E van de, Grol R: Interventions to improve management of anxiety disorders in general practice: a systematic review. $\mathrm{Br} J \mathrm{Gen}$ Pract 2005, 55:867-73.

15. Tylee $A$, Walters $P$ : Underrecognition of anxiety and mood disorders in primary care: why does the problem exist and what can be done? JClin Psychiatry 2007, 68(Suppl 2):27-30

16. Kessler D, Lloyd K, Lewis G, Gray DP: Cross sectional study of symptom attribution and recognition of depression and anxiety in primary care. BMJ 1999, 318(7181):436-39.

17. Rollman BL, Belnap BH, Mazumdar S, Zhu F, Kroenke K, Schulberg HC Shear MK: Symptomatic severity of Prime-MD diagnosed episodes of panic and generalized anxiety disorder in primary care. J Gen Intern Med 2005, 20(7):623-28

18. Stein MB, Sherbourne CD, Craske MG, Means-Christensen A, Bystritsky A Katon W, Sullivan G, Roy-Byrne PP: Quality of care for primary care patients with anxiety disorders. Am J Psychiatry 2004, 161(12):2230-37.

19. Ministry of Health, Diagnosis and Treatment Guideline for Primary Care. 2003:235-239. [in Turkish]
20. Ozcan M, Uguz F, Cilli AS: The prevalence of generalized anxiety disorder and comorbidity among psychiatric outpatients. Turk Psikiyatri Derg 2006, 17(4):276-85

21. Mergl R, Seidscheck I, Allgaier AK, Möller HJ, Hegerl U, Henkel V: Depressive, anxiety, and somatoform disorders in primary care: prevalence and recognition. Depress Anxiety 2007, 24(3):185-95.

22. Culpepper L: Generalized anxiety disorder in primary care: emerging issues in management and treatment. J Clin Psychiatry 2002, 63(Suppl 8):35-42.

23. Harman JS, Rollman BL, Hanusa BH, Lenze EJ, Shear MK: Physician office visits of adults for anxiety disorders in the United States, 1985-1998. Gen Intern Med 2002, 17(3):165-72.

24. Weisberg RB, Dyck I, Culpepper L, Keller MB: Psychiatric treatment in primary care patients with anxiety disorders: a comparison of care received from primary care providers and psychiatrists. Am J Psychiatry 2007, 164(2):276-82.

25. Fernández A, Haro JM, Codony M, Vilagut G, Martínez-Alonso M, Autonell J, Salvador-Carulla L, Ayuso-Mateos JL, Fullana MA, Alonso J: Treatment adequacy of anxiety and depressive disorders: primary versus specialised care in Spain. J Affect Disord 2006, 96(1-2):9-20

26. Tiemeier H, de Vries WJ, van het Loo M, Kahan JP, Klazinga N, Grol R, Rigter $\mathrm{H}$ : Guideline adherence rates and interprofessional variation in a vignette study of depression. Qual Saf Health Care 2002, 11(3):214-8.

27. Peabody JW, Luck J, Glassman P, Jain S, Hansen J, Spell M, Lee M: Measuring the quality of physician practice by using clinical vignettes: a prospective validation study. Ann Intern Med 2004, 141(10):771-80

28. Peabody JW, Liu A: A cross-national comparison of the quality of clinical care using vignettes. Health Policy Plan 2007, 22(5):294-302.

\section{Pre-publication history}

The pre-publication history for this paper can be accessed here: http://www.biomedcentral.com/1471-2296/11/30/prepub

doi: $10.1186 / 1471-2296-11-30$

Cite this article as: Kartal et al., Recognizing and managing anxiety disorders in primary health care in Turkey BMC Family Practice 2010, 11:30

\section{Submit your next manuscript to BioMed Central and take full advantage of:}

- Convenient online submission

- Thorough peer review

- No space constraints or color figure charges

- Immediate publication on acceptance

- Inclusion in PubMed, CAS, Scopus and Google Scholar

- Research which is freely available for redistribution 